\title{
DIAGNOSTIC SYSTEMS PLAN FOR THE ADVANCED LIGHT SOURCE TOP-OFF UPGRADE*
}

\author{
Walter Barry, Mike Chin, David Robin, Fernando Sannibale, Tom Scarvie, Christoph Steier, \\ LBNL, Berkeley, CA 94720, U.S.A.
}

\begin{abstract}
The Advanced Light Source (ALS) will soon be upgraded to enable top-off operations [1], in which electrons are quasi-continuously injected to produce constant stored beam current. The upgrade is structured in two phases. First, we will upgrade our injector from $1.5 \mathrm{GeV}$ to 1.9 $\mathrm{GeV}$ to allow full energy injection and will start top-off operations. In the second phase, we will upgrade the Booster Ring (BR) with a bunch cleaning system to allow high bunch purity top-off injection. A diagnostics upgrade will be crucial for success in both phases of the top-off project, and our plan for it is described in this paper. New booster ring diagnostics will include updated beam position monitor (BPM) electronics, a tune monitoring system, and a new scraper. Two new synchrotron light monitors and a beam stop will be added to the booster-tostorage ring transfer line (BTS), and all the existing beam current monitors along the accelerator chain will be integrated into a single injection efficiency monitoring application. A dedicated bunch purity monitor will be installed in the storage ring (SR). Together, these diagnostic upgrades will enable smooth commissioning of the full energy injector and a quick transition to high quality top-off operation at the ALS.
\end{abstract}

\section{INTRODUCTION}

The diagnostics in the BR and BTS transfer line will play a major role during the commissioning and operation of top-off mode at the ALS. Injection efficiency and short and long term stability of the BR operational conditions are the fundamental requirements for proper operation in this new mode.

The BR is currently operated with sextupole magnets off and without any orbit monitoring or control. The operators compensate for long-term drifts by making empirical readjustments. In top-off operation, tolerances on injection parameter fluctuations will be much tighter and will require more efficient diagnostics systems. For example: synchro-betatron resonance control is required during energy ramping, closed orbit and current monitoring are necessary for preserving injection efficiency, and bunch cleaning requires accurate control of tunes, chromaticities and orbit.

The BTS will be upgraded with two new synchrotron light monitors and a beam stop for tuning, a beam transmission monitor for the entire injector chain will be created, and a dedicated bunch purity monitoring system will be installed in the SR.

*Work supported by the Director, Office of Science, of the U.S. Department of Energy under Contract No. DE-AC03-76SF00098.

\section{BOOSTER RING}

\section{Orbit Monitoring}

The BR has 32 BPMs distributed along its circumference, equipped with original ALS electronics that allow for both single and multi turn measurements. Since the larger of the BR betatron tunes is less than seven, 13-14 BPMs will be sufficient for sampling the orbit in the booster. The existing 13 year old single turn electronics will be refurbished and, in the second phase of the project, modern multi turn electronics will be purchased to complete the upgrade of the BPM system.

The BPMs will have two functions for top-off operations. During commissioning, single turn or few turn measurements will be helpful for establishing circulating beam and critical for dynamics studies. Once routine topoff operations are established, an orbit measurement and feedback system will improve injection and bunch cleaning efficiency. A high level application, similar to the one existing for the ALS SR, will be developed to monitor and control the orbit in the BR.

\section{Bunch Cleaning}

Four weeks per year are dedicated to "two-bunch" operations at the ALS, in which two diametrically opposed buckets are filled with relatively high currents of about $30 \mathrm{~mA} / \mathrm{bunch}$. The $328 \mathrm{~ns}$ delay between these bunches is large enough to enable experiments with long relaxation times. The "two-bunch" user community is continuously growing and the scientific quality of their work is very high, so ensuring bunch purity during top-off operation is a high priority.

Our requirement on bunch purity is that the number of undesired electrons in the nominally empty buckets is kept to a level below $\sim 0.01 \%$ of the number of electrons in the main two bunches. Several mechanisms during the injection process can contribute to contamination, and even when injection is well tuned, contamination can still reach levels of $0.1 \%$, necessitating a bunch cleaning procedure.

Presently, bunch cleaning is performed in the SR after every injection and energy ramp to $1.9 \mathrm{GeV}$. The beam impedance of the ALS vacuum chamber induces betatron tune shift with current amplitude, so the small-current parasitic bunches have a tune significantly different from that of the high-current main bunches. Exciting the beam at the betatron frequency of the parasites generates large transverse oscillations of these undesired bunches with minor effects on the main bunches. A scraper is inserted far enough to clean out the oscillating particles. Unfortunately, the whole procedure requires about four 
minutes and perturbs the "good" bunches, making it incompatible with top-off operation. During the first phase of the upgrade, we will not use top-off injection during "two-bunch" operations and will continue to use the existing SR cleaning system.

In the second phase, we will adopt the convenient solution of cleaning in the BR before injecting into the SR. The planned scheme is based on the idea of resonantly exciting large transverse oscillations in all of the populated buckets, except for the ones we want to preserve $[2,3]$. Two signals are mixed together as shown in Figure 1.

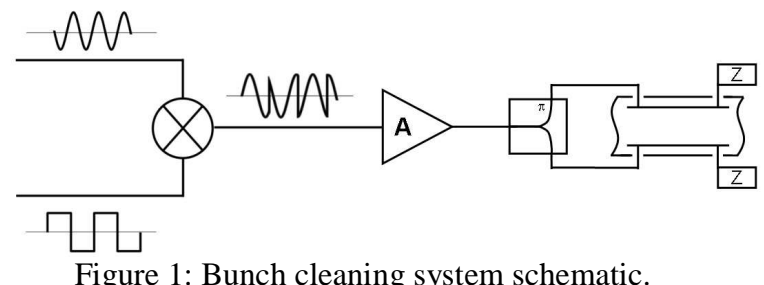

A sinusoidal wave at the frequency of one of the betatron sidebands is mixed with a square-like wave that switches polarity synchronously with the transit of the bunches to be saved, producing a signal which remains excited at the betatron tune but has zero amplitude coincident with the passage of the "good" bunches. The signal is amplified and excites the BR beam via a transverse kicker, with a scraper inserted in the plane of the excitation increasing the cleaning efficiency and reducing the power requirement for the amplifier. A trade-off between efficient cleaning and efficient injection into the BR will determine the final position of the scraper.

This scheme does require that no contamination occur during injection from the BR into the SR. The technique is already in routine operation at Spring 8 [4] in Japan and under final test at the ESRF [5] in France, with good results. At the ALS, we have successfully tested the complete cleaning procedure in the SR.

The cleaning system parameters were evaluated according to the criteria presented in reference [3]. Cleaning will be tuned for maximum efficiency at a beam energy of $300 \mathrm{MeV}$, with the vertical plane excitation applied for a few tens of milliseconds around this point in the energy ramp. Ideally, the chromaticity of the BR should be corrected to zero to avoid tune spread as the energy changes, or else more excitation power will be needed to maintain the required oscillation amplitude within the beam energy spread range.

\section{Kicker system}

The kicker for BR bunch cleaning will be a copy of the SR vertical transverse feedback system kicker: a vertical stripline pair with $30 \mathrm{~cm}$ long electrodes, each subtending $120 \mathrm{deg}$ of the beam. Driven differentially, the calculated transverse shunt impedance of the kicker at low frequencies is $8938 \mathrm{ohms}$, which compares favorably to bench measurements of about $10 \mathrm{k} \Omega$.
The azimuthal position of the vertical kicker in the ring will be as close as possible to the central defocusing quadrupole of the cell, where the vertical beta function has a local maximum. The power amplifiers for driving the kicker will be identical to the storage ring transverse feedback system amplifiers: 250 watt, $250 \mathrm{MHz}$ instantaneous bandwidth RF power amplifiers.

Nominally, one would use two amplifiers to drive the electrodes separately at opposite phases with a total available power of 500 watts. Recently, a bunch cleaning experiment was performed in the ALS SR at $1.9 \mathrm{GeV}$ using the transverse feedback amplifiers and kickers. In this case, only one electrode was driven with one amplifier, halving the effective shunt impedance and resulting in a $\sqrt{2}$ reduction in kick. A $125 \mathrm{MHz}$ sine wave, modulated by a vertical betatron tune line, was used to clean every other bunch in the ring. For 100 watts of drive power and nominal scraper depths, perfect bunch cleaning (within the approximate $0.1 \%$ resolution of our instrumentation) was practically instantaneous, as opposed to the present technique that takes several minutes.

Depending on the final position of the BR scraper during top-off operation, a more economical single electrode drive scheme may be adequate for cleaning. The current plan is to buy and install two amplifiers for the kicker, potentially operating only one and reserving the other as an online spare. A commercial function generator will replace the $125 \mathrm{MHz}$ sine wave used in the experiment to allow for general bunch cleaning.

\section{Vertical Scraper}

In the second phase of the project, a single blade vertical scraper, like the one presently installed in the ALS SR, will be installed in the BR for use in the cleaning system. The azimuthal position of the scraper in the ring will be as close as possible to the central defocusing quadrupole of the cell (cell symmetry point) where the vertical beta function has a local maximum.

\section{Tune Measurement}

In the first phase of the top-off upgrade, the betatron tunes in the BR will be measured by exciting the beam with the extraction kicker and Fourier analyzing the resulting signal from a BPM. Residual coupling will allow tune measurements in both planes.

In the second phase, accurate tune measurements will be required for bunch cleaning, so we will install a dedicated tune measurement system in the BR. A fast broadband FFT oscilloscope will extract the tune information from a BPM signal. The bunch-cleaning kicker and amplifier will excite the vertical tunes, and an additional kicker and amplifier will be installed for the horizontal plane. The azimuthal position of the horizontal kicker in the ring will be as close as possible to the last focusing quadrupole before the cell symmetry point, where the horizontal beta function assumes a local maximum. 


\section{BTS TRANSFER LINE}

\section{Synchrotron Radiation Monitors}

Two synchrotron radiation monitors in the BTS will non-destructively monitor the beam in the final part of the injection chain. The monitors will exploit the visible and infrared portion of the synchrotron radiation emitted in two of the four 20 degree dipoles in the BTS. The monitor with higher dispersion and low horizontal beta function at source point 1 (see Figure 2) will be installed during phase one to identify energy variations in the beam extracted from the BR. The system at the less-dispersive source point 2 will be installed during the second phase and will be better for beam emittance measurements.

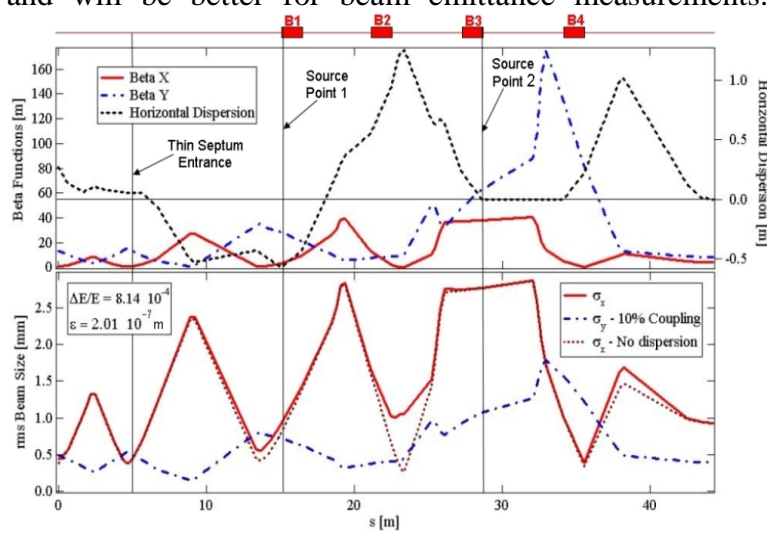

Figure 2: BTS optical function, beam sizes and SR source point positions.

A quick glance at the two monitors will indicate the health of the injector chain. The ESRF in Grenoble has a similar scheme in operation with good results [6].

\section{BTS Beam Stop}

During phase one, we will install an extractable beam stop at the end of the BTS transfer line, just before the first injection septum of the storage ring. This will allow for complete tuning of the injection chain without perturbing the SR beam.

\section{Beam Transmission Monitor}

The ALS complex has nine beam charge monitors: the linac (LN) and the linac-to-booster transfer line (LTB) are equipped with three, the BR has two, the BTS three, and there is one in the SR. The output of all these monitors will be displayed on a single high level software application, created during phase one, to provide a complete picture of the beam transmission efficiency along the injection chain.

\section{STORAGE RING}

During two-bunch operation, bunch purity in the SR must be measured. Historically, our digital scope measurement of a BPM signal resolved bunch contamination of a few parts per thousand. In the second phase of the top-off upgrade, we will install a dedicated purity monitor with a higher resolution $\left(<10^{-4}\right)$ that will provide better diagnosis of the bunch cleaning procedure.
The proposed system is based on a photon counting scheme; the block diagram of the system is shown in Figure 3.

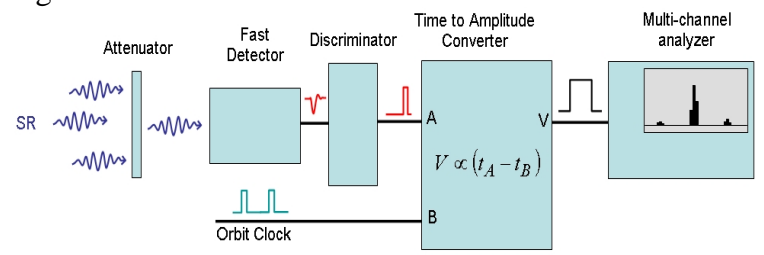

Figure 3: Bunch purity monitor block diagram.

The attenuation should be strong enough to get into the single-photon count regime, in which less than one photon on average is detected for every passage of the bunch. This is needed to avoid photon pile-up and to ensure a linear system response.

One decision that remains is whether to use visible photons or X-rays for photon counting. The detector must be fast enough to discriminate between contiguous buckets $(2 \mathrm{~ns})$ at a resolution smaller than $10^{-4}$. This demand is at the limit of what can be achieved with photomultipliers using visible light, but it is well within the capability of x-ray detectors such as avalanche photo diodes. On the other hand, the visible option is simpler because it could use the synchrotron radiation already available from the ALS diagnostic beam lines 3.1 or 7.2, while for the $\mathrm{x}$-ray case a new port with a thin beryllium window would be required to access the x-rays.

\section{CONCLUSION}

When the ALS injector is converted into a full $1.9 \mathrm{GeV}$ system for top-off operations, the demands on it will expand significantly. The orbit, injector beam characteristics, and injection efficiency will need to be monitored and tightly controlled. High purity two-bunch operations will be maintained by changing from a SRbased cleaning scheme to one in which the bunches are purified during acceleration in the $\mathrm{BR}$, and installing a higher resolution SR bunch purity monitor. All these upgrades will work together to ensure that top-off operations at the ALS commission smoothly and work reliably, so we can continue to provide high quality beam for our users.

\section{REFERENCES}

[1] D. Robin, RPAE063, Particle Accelerator Conference, Knoxville, US, May 2005.

[2] N. Sereno, Proceedings of the 1999 Particle Accelerator Conference, New York, US, 1999, p. 2322.

[3] H. Suzuki, et al., Nuclear Instruments and Methods in Physiscs Research A 444 (2000) 515-533.

[4] T. Aoki, et al., Proceedings of the 2003 Particle Accelerator Conference, Portland, US, May 2003, p. 2551.

[5] E. Plouviez, ESRF Machine Technical Note 0104/MDT.

[6] E. Plouviez, P. Ellaume, private communications. 